\title{
On the origin of microwave type U-bursts
}

\author{
A. T. Altyntsev ${ }^{1}$, A. A. Kuznetsov ${ }^{1}$, N. S. Meshalkina ${ }^{1}$, and Yan Yihua ${ }^{2}$ \\ ${ }^{1}$ Institute of Solar-Terrestrial Physics, Siberian Branch of the Russian Academy of Sciences (ISTP SB RAS), PO Box 4026, \\ Irkutsk 33, 664033, Russia \\ e-mail: altyntsev@iszf.irk.ru \\ 2 National Astronomical Observatories, Chinese Academy of Sciences, Datun Road A20, Chaoyang District, \\ Beijing 100012, PR China \\ e-mail: yyh@bao.ac.cn
}

Received 8 April 2003 / Accepted 1 August 2003

\begin{abstract}
An analysis is made of the observations of U-type cm-bursts recorded simultaneously with high spatial (Siberian solar radio telescope) and spectral resolution (National Astronomical Observatories/Beijing spectropolarimeters). It is shown that the source positions of increasing and decreasing branches of the U-burst coincide within a few arcsec. The suggestion is made that the occurrence of the observed type U-bursts at microwave frequencies is associated with a pressure and density response to rapid heating pulses in flare loops.
\end{abstract}

Key words. Sun: flares - Sun: activity - Sun: radio radiation

\section{Introduction}

It is commmonly accepted that the fine structure of narrowband microwave bursts in dynamic spectra reflects a plasma density variation in the emission source. Type U-bursts, and more complicated $\mathrm{N}$-shaped bursts, constitute a particular kind of type III bursts. It is believed that type III bursts trace the propagation of electron beams along magnetic field lines in the solar corona (Wild \& Smerd 1972; Caroubalos et al. 1987). Electrons with a velocity of about $10^{10} \mathrm{~cm} / \mathrm{s}$ guided along magnetic field lines excite Langmuir waves which are transformed to electromagnetic waves near the local plasma frequency and its harmonic by nonlinear plasma mechanisms (Melrose 1987). On the dynamic spectrum they are seen as rapidly drifting narrow band emission. When electrons are accelerated along closed magnetic field lines (in a magnetic arch), the radio burst appears as the inverted letter U: the turnover point with a minimum frequency corresponds to the top of the arch. Such bursts are frequently observed in meter and decimeter radio emission (Maxwell \& Swarup 1958; Caroubalos et al. 1987).

Indirect estimates of the velocities of electron beams generating type III bursts, based on analyzing frequency drift velocities, were made in a large number of publications. For a wide frequency range they are estimated at $0.15 c$ ( $c$ is the velocity of light), corresponding to the energy of about $6 \mathrm{keV}$ (see, e.g., a review by Aschwanden 2002).

In this model the frequency of the U-burst is directly determined by the plasma density value in the U-burst

Send offprint requests to: $\mathrm{N}$. S. Meshalkina,

e-mail: nata@iszf.irk.ru emission region. For the cm-range the plasma density is so large that the collision deflection time of the beam electrons becomes too short in comparison with the pulse duration. On the other hand, observations of subsecond pulse sources with high temporal and spatial resolution were available in microwaves. Regrettably, these telescopes recorded the emission at a single frequency, and dynamic spectra of pulses were unknown.

Observations with the multi-beam antenna at $48 \mathrm{GHz}$ showed that the subsecond structures were produced in small areas with the size of about 3 arcsec, near the instrumental limit (Correia et al. 1995). These results are in agreement with BIMA observations, which showed that for certain bursts at $86 \mathrm{GHz}$ the dominant source sizes were about a few arcseconds (Lim et al. 1992).

SSRT observations with a spatial resolution of 15 arcsec showed that the source sizes of subsecond pulses at $5.7 \mathrm{GHz}$ are below the spatial resolution and do not exceed the value 5 arcsec in the central part of the solar disk, where the scattering of emission along its path through the corona is low (Altyntsev et al. 1996).

Measurements of spatial characteristics of drifting burst sources in the microwave range were made possible by the combined analysis of the data from the SSRT and from the NAOC spectropolarimeter operating in the frequency range 5.2-7.6 GHz (Altyntsev et al. 2003). They are characterized by millisecond temporal resolution, since the duration of type III bursts in the microwave range does not exceed one second (Bruggmann et al. 1990). The distinguishing feature of the SSRT 1d-mode observations is the possibility of obtaining the spatial structure of drifting bursts at two nearby frequencies 
simultaneously. Based on studying several tens of bursts with the drift velocity ranging from $-8 \mathrm{GHz} / \mathrm{s}$ to $20 \mathrm{GHz} / \mathrm{s}$, it was shown that the difference in the position of the sources, the emission frequency of which differed by $80 \mathrm{MHz}$, did not exceed 3 arcsec in magnitude and was independent of the value and sign of the drift velocity (Meshalkina et al. 2002).

Of special interest is the study of the dynamics of U-type burst sources in which one would expect a significant difference in the position of sources having an identical emission frequency but referring to different branches of the U-burst. The displacement depends on the loop size, and can be spatially resolved with SSRT observations. Such bursts in a band around the SSRT receiving frequency of $5.7 \mathrm{GHz}$ were observed by Wang et al. (2001). The spatial displacement was first measured for the U-burst in the September 17, 2001 flare (Sych et al. 2002; Altyntsev et al. 2003). Contrary to the above suggection, it was found that the displacement did not exceed a few arcsec.

The objective of this paper is to study a number of U-type events that recorded simultaneously with the SSRT and with the NAOC spectropolarimeter to date.

\section{Observing techniques}

The Siberian Solar Radio Telescope (SSRT), is a crossed radio interferometer, consisting of two lines of antennas: east-west (EW), and north-south (NS), operating in the frequency range 5.67-5.79 GHz (Smolkov et al. 1986; Grechnev et al. 2003). Radio maps of the full solar disk were recorded at intervals of 3-5 min. The cadence of the two-dimensional imaging is too low to study subsecond events. Two-dimensional images were used to align the SSRT images and maps in other emissions. Investigations of the fine temporal structure of flare bursts are based on using data recorded by the EW and NS arrays separately which provide one-dimensional images (scans) of the solar disk.

Methods for analyzing one-dimensional solar images were described by Altyntsev et al. (1996, 2003). The SSRT receiving system comprises the spectrum analyser in the $120 \mathrm{MHz}$ frequency band which is represented by the acousto-optic receiver featuring 250 frequency channels which correspond to the fans of knife-edge beams for the NS and EW arrays, respectively. The bandwidth of one frequency channel is $0.48 \mathrm{MHz}$. The response at each frequency corresponds to the emission from a narrow strip on the solar disk whose position and width depend on the frequency. This dependence is determined by the time of observation, and the type of array. With the small size of microwave burst sources, they are recorded by the SSRT in a narrow band not exceeding $5 \mathrm{MHz}$.

Signals from all channels are recorded simultaneously and generate a one-dimensional distribution (scan) of solar radio brightness every $14 \mathrm{~ms}$. The components of the circular polarization $(\mathrm{R}$ and $\mathrm{L})$ are recorded successively within the interval of $7 \mathrm{~ms}$ duration each. The beam widths were down to 15 arcsec for one dimensional scans, and depend on the array and local time of observation. The crossing of the corresponding EW and NS strips points at the source of the subsecond pulse in the SSRT two-dimensional map. A distinctive feature of some events was the simultaneous detection of the same microwave burst in two frequency bands of the SSRT interferometer (Altyntsev et al. 2003).

To study the relative time variations of the source position we used the variations of the "gravity" centre of parts of the scan where the subsecond sources were located. To remove the influence of the background burst on the SSP scans, the scan before the subsecond pulse was extracted from them. A modeling of this procedure with a corresponding noise level has shown that the accuracy of relative centroid positioning was about 2 arcsec. A two-dimensional full radio map at the time close to the subsecond burst, was used to align the microwave images with the MDI magnetogram, or with other emission maps. The effect of the Sun rotation was taken into account. We believe that the accuracy of aligning was not worse than 10 arcsec.

Dynamic spectra were observed with the Solar Radio Broadband Fast Dynamic Spectrometers (5.2-7.6 frequency range) at Huairou Solar Observing Station of the National Astronomical Observatories ( $\mathrm{Fu}$ et al. 1995). The reception band of an individual frequency channel of the NAOC spectropolarimeter is $20 \mathrm{MHz}$, and the temporal resolution is $5.9 \mathrm{~ms}$.

To study flare structures we used the data of soft and hard X-rays from the Yohkoh satellite, MDI magnetograms, and images of the Sun from the Nobeyama radioheliograph at $17 \mathrm{GHz}$. Also, Yohkoh/HXT time profiles were used in the comparison with the SSRT time profiles.

\section{Observations}

The U-type events are very rare in the microwaves. In 80 events with subsecond structures we have found four drifting U-type bursts, the branches of which intersected the SSRT reception band on the dynamic spectra, and the turnover frequency was less than $5.7 \mathrm{GHz}$.

\section{June 2, 2000}

A drifting N-type burst (see Fig. 1) was recorded at the early stage of the M1.2 flare in the AR 9026 (N17E75) located near the east limb. X-ray emission at the time of the N-burst could be estimated as relatively soft, because Yohkoh/HXR had not yet switched on the flare mode of observation.

The pulse was observed in the frequency range 5.5$5.95 \mathrm{GHz}(\Delta f=0.45 \mathrm{GHz})$, a total duration $\tau_{\text {tot }} \approx 0.5 \mathrm{~s}$, and the peak flux $8 \mathrm{sfu}$. The drift velocities of the branches of the $\mathrm{N}$-shaped structure were $2 \mathrm{GHz} / \mathrm{s} ;-4.4 \mathrm{GHz} / \mathrm{s}$, and $1.2 \mathrm{GHz} / \mathrm{s}$, respectively. The instant width of pulse emission $\Delta f_{\text {ins }}$ was less than one percent of the receiving frequency, but about half of the pulse total bandwidth.

Further, we consider the last two branches between the vertical lines in the bottom panel to be the U-shaped structure in this event. The time interval between the times of recording the SSRT signals of the decreasing and increasing branches of the U-structure with the NS array is $\tau_{\text {ssrt }}=220 \mathrm{~ms}$. The subsecond burst was simultaneously observed with the two linear interferometers of SSRT: EW at $5.68 \mathrm{GHz}$, and NS at $5.73 \mathrm{GHz}$ (Fig. 2). Time profiles of signals from the corresponding 

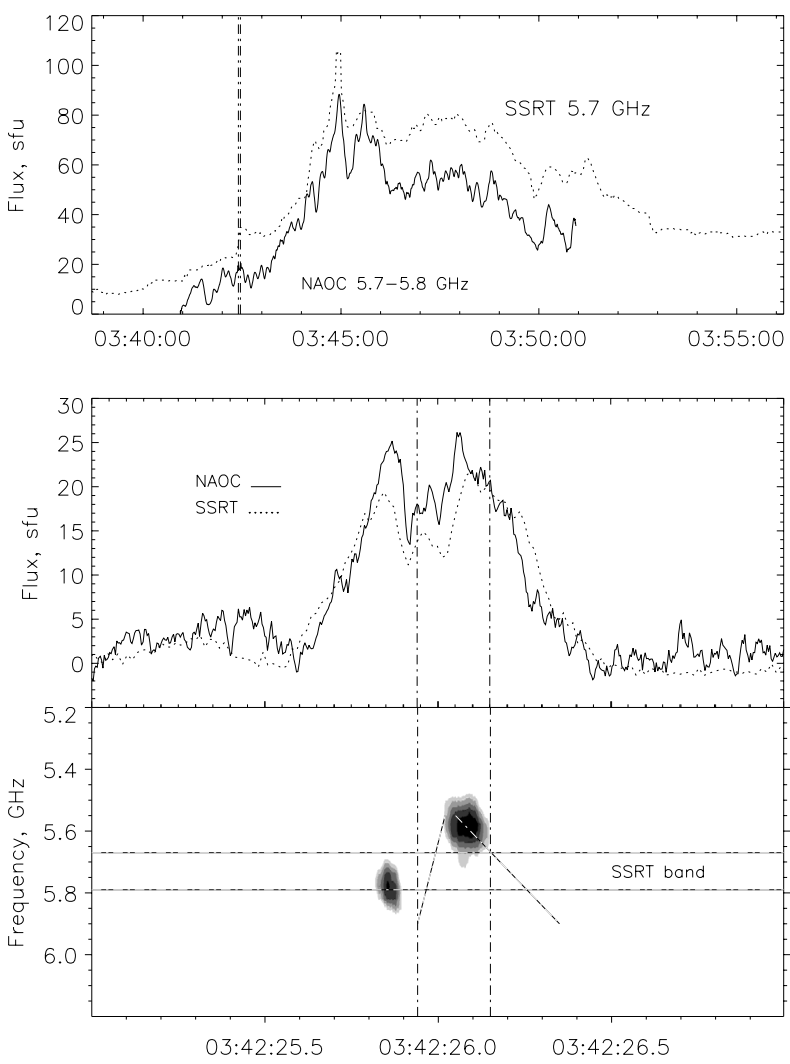

Fig. 1. June 2, 2000. Time profiles of the microwave burst (top), extended time profiles for the interval marked by the vertical lines in the upper panel (middle), and the dynamic spectrum (using a nonlinear color table, bottom).

frequency channels of the NAOC and SSRT spectropolarimeters are similar, which suggests the solar origin of the pulses and the small influence of noise. Noteworthy are some differences of the time profiles at the EW and NS frequencies differing by $50 \mathrm{MHz}$, or less than $1 \%$ of the receiving frequency. All branches of the burst have a significant degree of circular polarization of the same sign, i.e. left-handed (up to 50\%).

The source position of the drifting burst relative to the background burst in different emission branches is shown in Fig. 3. The main polarized source is seen at $5.7 \mathrm{GHz}$ and $17 \mathrm{GHz}$. The second source can be distinguished northeastward in the image at $17 \mathrm{GHz}$ where spatial resolution is higher. The subsecond pulse was located in this background source, as is seen from the crossing of the dash-dotted lines. Note, on the one hand, that the source of the subsecond burst is localized at some distance from accelerated electron precipitation sites seen as two sources in hard X-ray emission. On the other hand, it corresponds to the western point of the strip in soft X-ray emission corresponding to an increased emission measure. The estimate of the soft X-ray emission measure is available a minute after the subsecond pulse and it is on the order of $10^{11} \mathrm{~cm}^{-3}$ under a reasonable assumption about the size of dense region.

The flare was near the east limb, and the microwave source of the background burst was above the spots of the bipolar active region (Fig. 4). The subsecond source was located higher than the background burst. Its height was close to 20 arcsec above the spots.
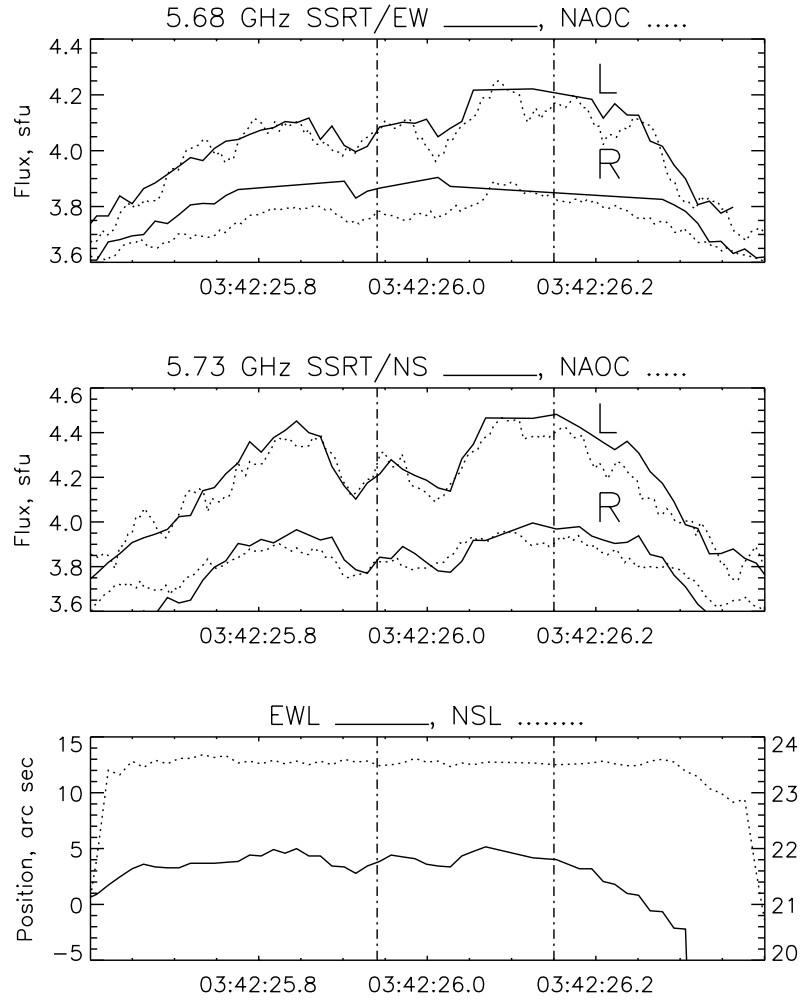

Fig. 2. June 2, 2000. Expanded time profiles of flux: EW linear interferometer at $5.68 \mathrm{GHz}$ for the left- and right-handed circular polarizations (top), NS at $5.73 \mathrm{GHz}$ frequency (middle); plots the centerof-gravity positions of one-dimensional scans versus time (bottom). There are shown the positions of the left-hand polarized emission components relative to the background burst's centroid. Vertical lines correspond to the lines in the bottom panel of Fig. 1.

Figure 2 (bottom) plots the center-of-gravity positions of one-dimensional scans versus time. In this event the SSRT beam widths are $16.4 \operatorname{arcsec}(\mathrm{EW})$ and $17.3 \operatorname{arcsec}(\mathrm{NS})$. It is evident that along both directions of scanning the displacement of the source does not exceed 3 arcsec throughout the entire interval with the $\mathrm{N}$-structure, including the times of recording the U-burst branches. This displacement is about the accuracy of brightness centroid positioning in the one-dimension mode.

March 30, 2001

The fine structures of the flare (M2.2, 05:09 UT, AR 9393, N18W18) were briefly outlined in a paper of Meshalkina et al. (2002). Several tens of subsecond type III bursts were observed in the flash phase of the impulsive flare. The mean drift velocity was $9.6 \pm 3.9 \mathrm{GHz} / \mathrm{s}$. There was no correlation of subsecond microwave pulses with the fine structure of hard X-ray emission. The pulses had a high degree of circular polarization (over $30 \%$ ) of left-hand sign.

The structure of microwave sources is shown in Fig. 5.

There is a bright source in intensity, right-handed polarized. The left-handed polarized source that has shifted eastward is well seen in the V-map. Subsecond pulses were observed only with the NS interferometer in this event. The figure shows that the knife-edge strip, corresponding to the brightness center of the subsecond burst source, crosses the main source of the background burst (top). The brightest patch of the loop seen in 
Table 1. Events with U-type pulses. Flux - pulse amplitude at the SSRT receiving frequency, $\Delta f$ - total width of the U-burst spectrum, $\Delta f_{\text {ins }}-$ instantaneous bandwidth, $\partial f / \partial t$ - drift velocities of different branches are shown sequentially, $\tau_{\text {tot }}-$ total duration, $\tau_{\text {sst }}-$ interval between the recording of the U-structure branches at the SSRT frequency.

\begin{tabular}{llllll}
\hline \hline Date & Time & Flux, sfu & $\Delta f / \Delta f_{\text {ins }}, \mathrm{GHz}$ & $\partial f / \partial t, \mathrm{GHz} / \mathrm{s}$ & $\tau_{\text {tot }} / \tau_{\text {ssrt }}, \mathrm{s}$ \\
\hline $2000 / 06 / 02$ & $03: 42: 26.2$ & 8 & $0.45 / 0.25$ & 2.0 & $0.45 / 0.22$ \\
& & & & -4.4 & \\
& & & & 1.2 & \\
$2001 / 03 / 30$ & $05: 12: 55.9$ & 0.1 & $1.0 / 0.22$ & -5.9 & $0.42 / 0.27$ \\
& & & & 5.9 & \\
$2001 / 03 / 30$ & $05: 13: 24.7$ & 0.1 & $0.7 / 0.3$ & -8.6 & $0.15 / 0.1$ \\
& & & & 7.5 & \\
$2001 / 09 / 17$ & $08: 20: 58.2$ & 180 & $0.2 / 0.05$ & -4.4 & $0.07 / 0.05$ \\
& & & & 7.0 & \\
\hline
\end{tabular}

the emission measure map (bottom), was calculated from soft $\mathrm{X}$-ray data. Thus it would appear reasonable that the bursts are generated in this patch with increased plasma density and the displacements in the scans correspond to displacements along the loop. The source positions of subsecond pulses lay near the brightness center of the background burst and generally were changing with a change in background burst position which was gradually displaced by $4-5$ arcsec for the period with the fine time structure (Meshalkina et al. 2002). The source of the background burst was well apart from the neutral line of the photosperic magnetic field (Fig. 4).

These fine structures included two weak pulses, the dynamic spectra of which had an U-like shape, and the frequency range made it possible to measure the source positions in different branches (see Figs. 6-7, the intervals with U-shaped bursts marked in the dynamic spectrum panels by vertical lines).

The parameters of these bursts are presented in Table 1. It should be noted that the events are small in magnitude, but the data can be considered reliable, since they are in agreement with independent measurements from SSRT and NAOC. In this event, the SSRT beam width is 25.9 arcsec. The displacements of the sources determined at the time of recording the U-burst branch do not exceed 3 arcsec and are on the order of the measurement error.

The observations of this flare were characterized by the simultaneous recording of the sources in two frequency ranges around $5.69 \mathrm{GHz}$ and $5.78 \mathrm{GHz}$. This mode of observation is described in detail in a paper of Altyntsev et al. (2003). Time profiles at these frequencies are in good agreement. It is seen that the difference in the positions at these frequencies does not exceed 4 arcsec, and there is a tendency to increase at the times of recording the branches.

\section{September 17, 2001}

Sych et al. (2002) and Altyntsev et al. (2003) reported a detailed analysis of the spatial structure of this event. Based on the results derived from analyzing the plasma parameters in the flaring region at the site of generation of the subsecond pulse (relatively high plasma density, and small magnitude of the magnetic field), Altyntsev et al. (2003) concluded that the emission of this U-shaped burst is generated at the frequency near the harmonic of plasma frequency.
A type U-burst (see Fig. 8) was recorded during the early phase of the M1.5 flare in the AR 9618 (S12E06) that occurred near the center of the solar disk.

The U-pulse duration was about $70 \mathrm{~ms}$. The subsecond pulse was left-hand circularly polarized, with the polarization reaching $30 \%$. The drift velocities of the U-shaped structure branches were $4.4 \mathrm{GHz} / \mathrm{s}$ and $7.0 \mathrm{GHz} / \mathrm{s}$, respectively. The interval between the points of passage of the U-shaped structure branches was $50 \mathrm{~ms}$ at $5.7 \mathrm{GHz}$. There was no correspondence with pulses in hard X-ray emission.

The pulses were observed with both linear interferometers at $5.69 \mathrm{GHz}$ and $5.77 \mathrm{GHz}(\mathrm{EW})$, and $5.71 \mathrm{GHz}(\mathrm{NS})$. The angle between their directions of scanning was 46.4 degrees, and the beam widths were 18.2 arcsec and $24.3 \operatorname{arcsec}$ for NS and $\mathrm{EW}$, respectively. A comparison of $2 \mathrm{~d}$ microwave maps with images in soft X-ray emission suggests that the main structural element of the flare which determines the microwave emission of the background burst is the loop connecting regions nearby magnetic spots (Fig. 4), seen as microwave sources with opposite circular polarization (Altyntsev et al. 2003). The pulse source was located near the loop top. The distance between the pulse source and the neutral line was about 20 arcsec. A relative referencing of the X-ray and microwave images shows that the SSP source is localized near the brightness maximum of X-ray emission, which corresponds to the region with high plasma density.

During the pulse, the position of the source did not change considerably along both scanning directions and in both polarization components. It is evident from Fig. 8 that for both linear interferometers the displacement of the sources does not exceed 3 arcsec for the times, corresponding to the recording of different branches of the U-structure. There is no distinct shift of the source positions obtained with the EW array at the time near the minimum of the U-structure at the neighboring frequencies.

\section{Discussion}

Table 1 summarizes the observations of four microwave bursts with the type U dynamic spectrum. The pulse amplitude at the SSRT receiving frequency is changed three orders of magnitude in the pulses used in the analysis. The total bandwidth of the pulses was within $0.2-1.0 \mathrm{GHz}$, averaging about $0.5 \mathrm{GHz}$, 

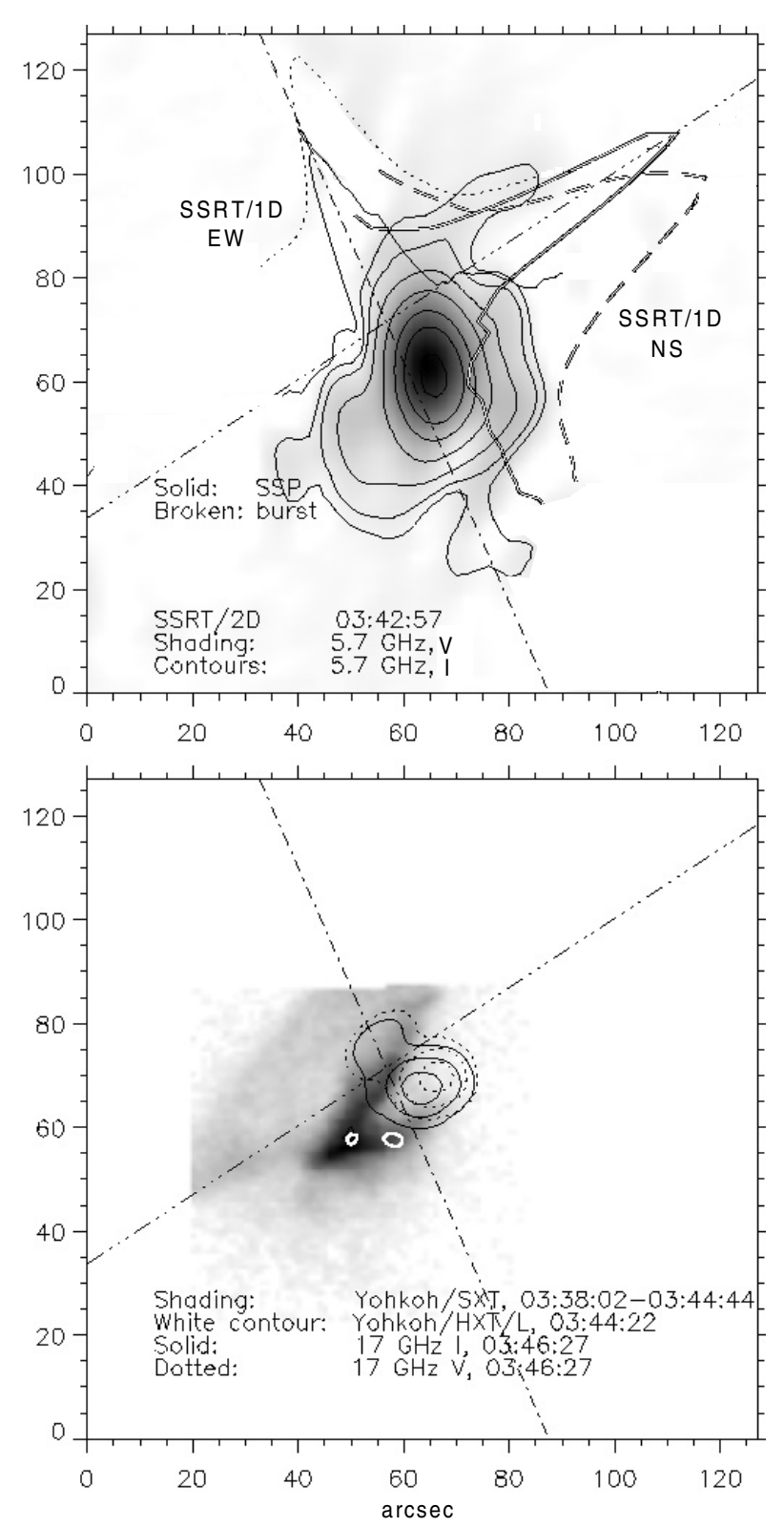

Fig. 3. June 2, 2000. Structure of flaring sources in soft and hard X-ray emissions together with microwave images at $17 \mathrm{GHz}$ in intensity and polarization (lower panel). One-dimensional and two-dimensional SSRT images at $5.7 \mathrm{GHz}$ (upper panel). The crossed straight lines show the direction of the strips, the emission of which is recorded by the EW and NS linear interferometers. To the point of intersection there corresponds the source position of the subsecond drifting burst.

or $10 \%$ of the mean emission frequency. The instantaneous emission bandwidth made up $1-5 \%$ and was about $10-50 \%$ of the pulse band. The drift velocity in different pulses differed by one order of magnitude in absolute value, within 1 and $10 \mathrm{GHz} / \mathrm{s}$. The total duration of the U-structures reached $0.5 \mathrm{~s}$, and the duration of the interval between the times of recording different branches varied from 50 to $270 \mathrm{~ms}$.

Direct estimates of the source sizes are not reliable, because their widths in the 1d-distributions were about the SSRT beam width. The accuracy of relative positioning using the center gravity method is about 2-3 arcsec. As was
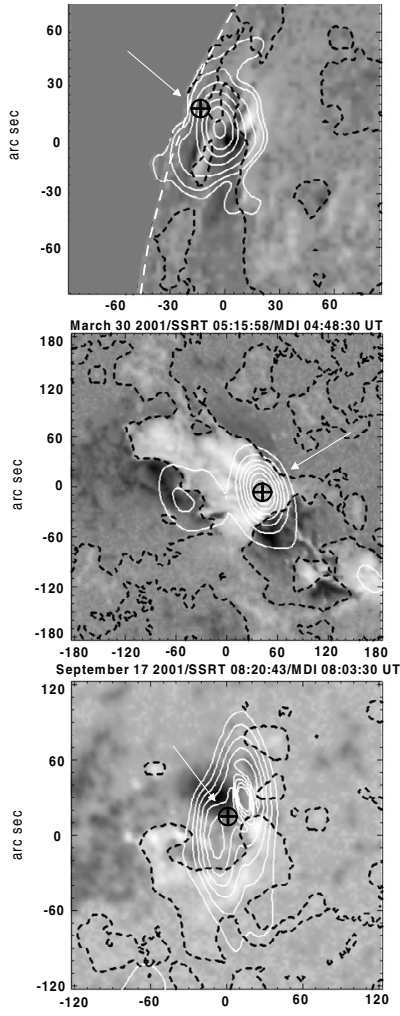

Fig. 4. Positions of the background burst sources (contours, I) and the subsecond sources (crosses in the circles) in the MDI magnitograms (shading), rotated for the burst time. Neutral lines of magnetic field are shown by dashed lines.

shown, the displacements of the source positions referring to different branches of U-shape structures do not exceed this value, or three thousand kilometers.

In three events when the sources could be observed simultaneously at two frequencies, it was possible to estimate the difference of their positions in each branch of the U-structure. The displacements of the sources with the emission frequency differing by $50-80 \mathrm{MHz}$ was also about a few thousand kilometers.

The polarization of the pulse emission was opposite to that of the background burst in all cases, and its sign was the same in both branches of the U-structure. There is no clear response to the subsecond pulses in hard X-rays in all events.

\subsection{The emission mechanism}

In publications devoted to the study of the time structure of narrow band microwave emission, the following sequence of reasoning is usual. From the narrowness of the instant emission band, we can conclude that the emission mechanism is a coherent one. Also, it is customary to suggest an anomalously high brightness temperature of the emission source. As there are no direct observations with sufficient spatial resolution, estimates of the source size (a few hundred kilometers) are based on the assumptions about the emission mechanisms, and, in particular, on the dependence of the emission frequency on plasma parameters in the source: plasma density, and the magnetic field. It is assumed that the coherent emission 

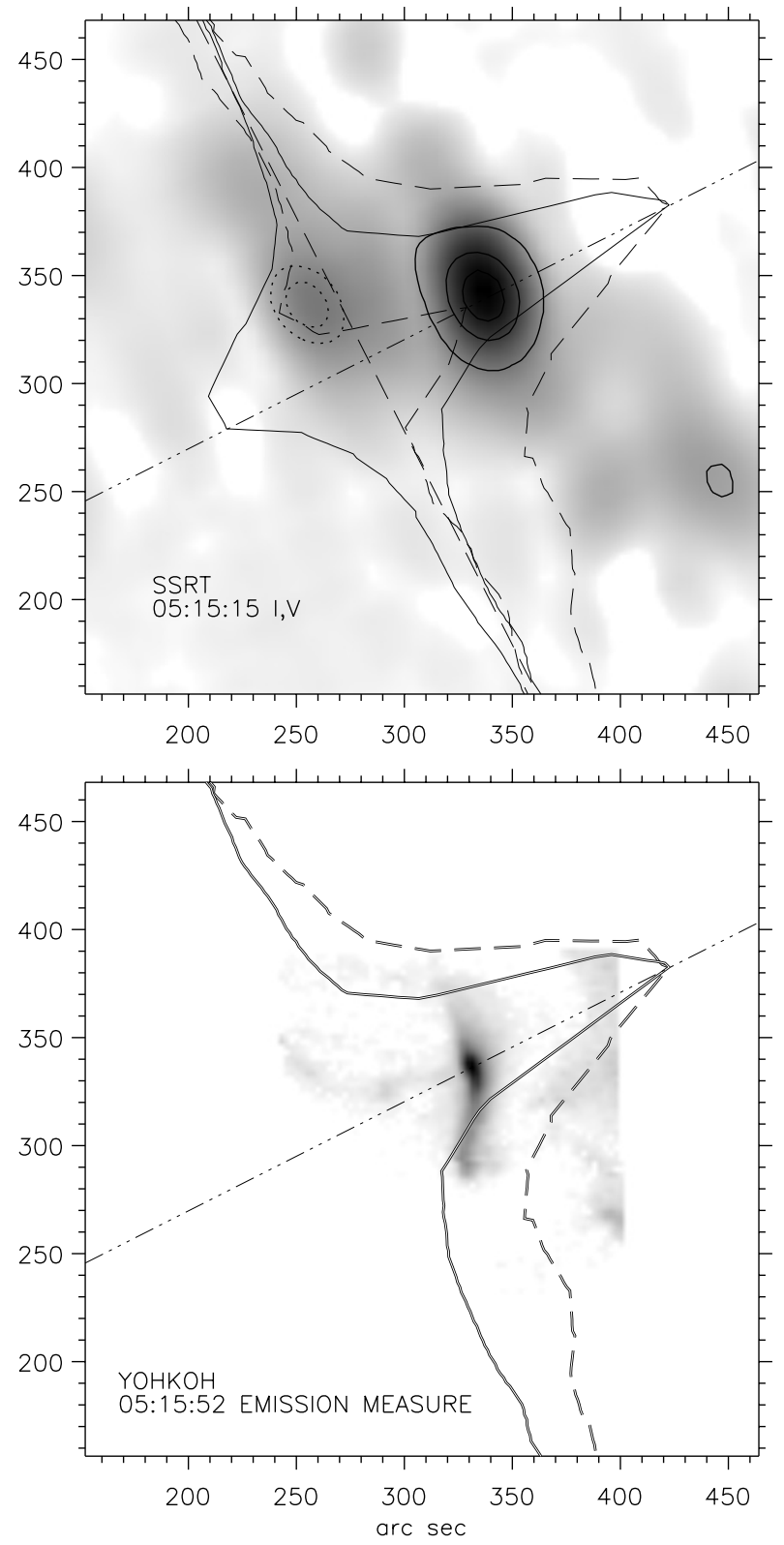

Fig. 5. March 30, 2001. Structure of microwave sources (top) in comparison with the soft X-ray emission measure map (bottom). Top - maps of brightness temperature (gray), polarization degree, 1ddistributions (I,V) of the background burst (dashed lines) and of subsecond pulse (solid).

frequency is close to the plasma frequency or its harmonics. In the microwave range, Langmuir emission and gyroresonance emission are considered.

Observations of subsecond pulses acquired with large interferometers $(5.7 \mathrm{GHz}, \mathrm{SSRT}$; $17 \mathrm{GHz}$, NoRH) made it possible to localize them in the flaring region and estimate the plasma parameters in their neighborhoods. It was shown that fine-structure sources tend to occur in the flare region with high plasma density sufficient for the generation of plasma frequency harmonic. With respect to cyclotron harmonics, the observed frequencies correspond to high harmonic numbers (higher than the sixth one), even if the magnitude of the magnetic field in the source at the photospheric level is used
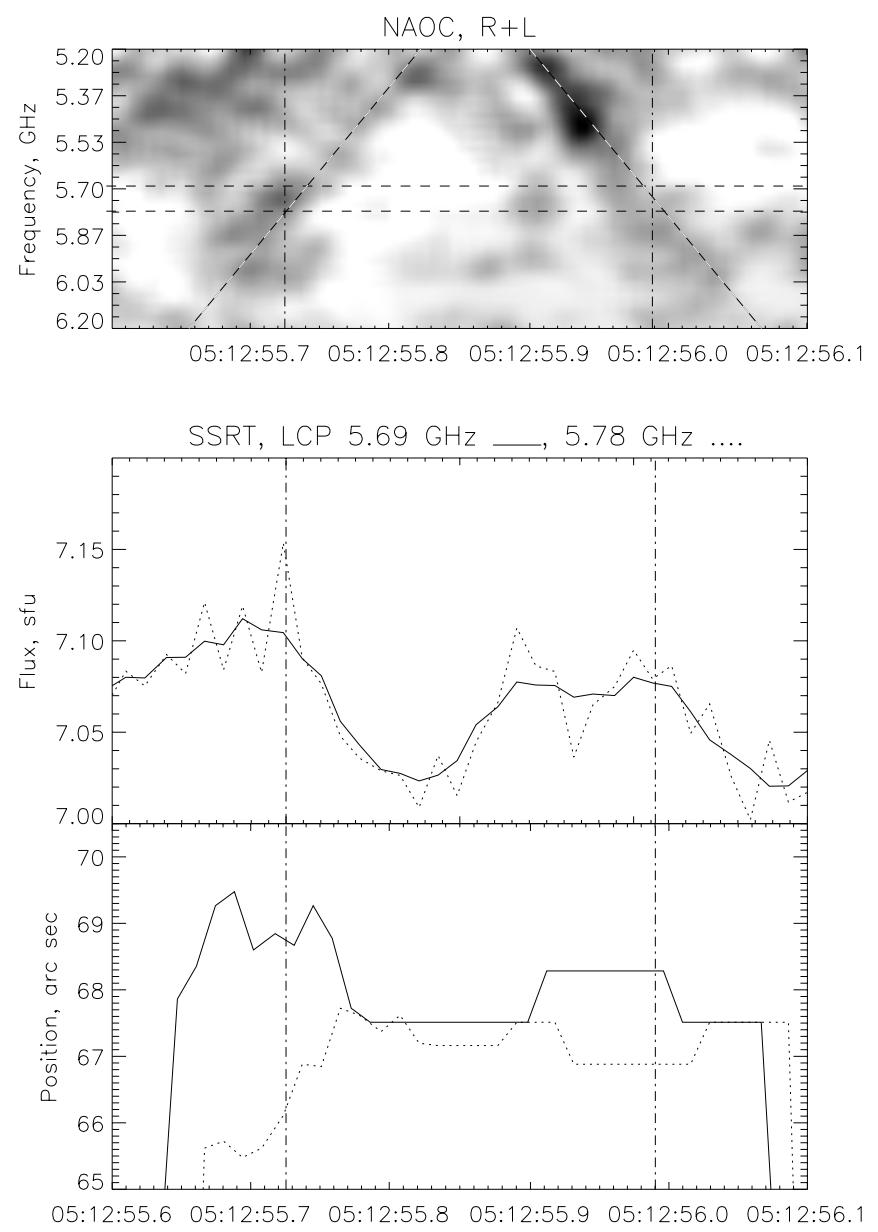

Fig. 6. March 30, 2001. Dynamic spectrum and time profile of the U-shaped burst at 05:12:55.9 UT.

in estimations. These numbers are too high for developing electron-cyclotron maser emission (Fleishman \& Melnikov 1998). Observations also indicate that the sources of narrow band subsecond pulses usually are far from sunspots with a strong magnetic field. This conclusion is also justified for the events with U-structures discussed in this paper.

It will therefore be assumed in what follows that the emission of U-structures is generated by a plasma mechanism, at the frequency close to the Langmuir frequency harmonic, $f=\frac{1}{\pi} \sqrt{\frac{4 \pi n e^{2}}{m}}$, where $n$ is plasma density, $e$ and $m$ are the electron charge and mass. For harmonic emission we have the more reasonable requirement for the value of plasma density (by a factor of four smaller than that at the fundamental frequency), and the free-free absorption is smaller by a factor of 16 (Dulk 1985). The estimate of the optical thickness of emission at the double Langmuir frequency $\tau \cong\left(\frac{T}{2 \mathrm{MK}}\right)^{-3 / 2}\left(\frac{h}{1000 \mathrm{~km}}\right)$ was obtained for the case of an exponential decrease in plasma density from the source with a scale $h$ outside of the source. It is evident that at usual coronal temperatures the optical thickness is on the order of unity for flaring magnetic loops. 

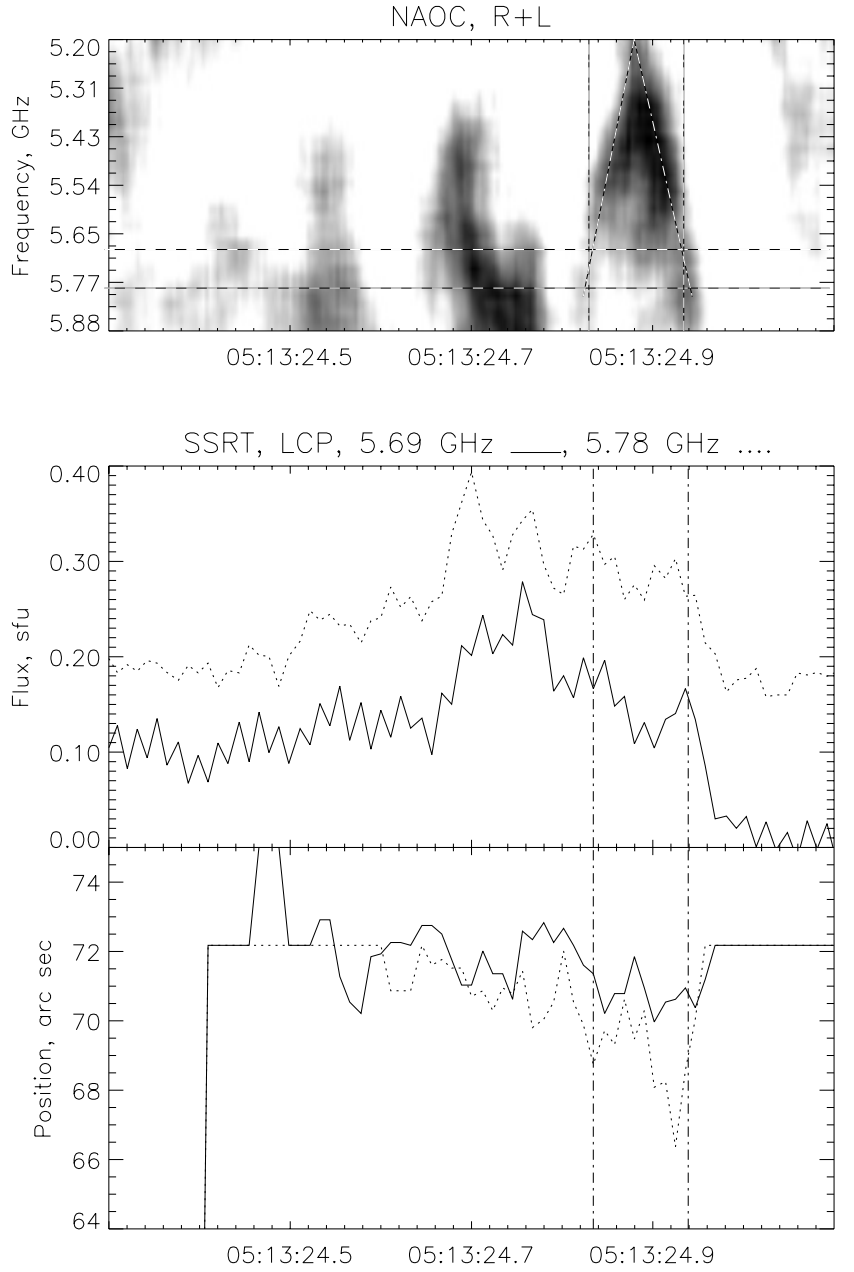

Fig. 7. March 30, 2001. Time profile and dynamic spectrum of the U-shaped burst at 05:13:24.9 UT.

\subsection{Kinematics of the emission region}

Suppose that the U-shape dynamic spectrum in microwaves appears due to an exciter moving along the flare loop. As follows from observations, the total length of the emitting region does not exceed three thousand kilometers around the loop top. Because the U-burst sources were located well apart from the neutral line of magnetic field (more than 10 arcsec for the events under study), their heights are not less than 10 thousand kilometers. It is known that the height of the subsecond source can reach a value of up to 30 thousand kilometers (Altyntsev et al. 1996).

On the other hand, bursts are observed in the frequency range from 5.2 to $6.2 \mathrm{GHz}$. For harmonic emission this range corresponds to plasma density variations in the source $(1 \pm$ $0.2) \times 10^{11} \mathrm{~cm}^{-3}$. If we take the observed source size $(\lambda=$ $3000 \mathrm{~km}$ ) as an upper limit of the hydrostatic density scale height along a magnetic field line we find a corresponding scale height temperature of $T=(1 \mathrm{MK}) \frac{\lambda}{47000 \mathrm{~km}}=6 \times 10^{4} \mathrm{~K}$. This temperature value is far too low for a flare loop, and thus suggests that the flare loop in which the microwave burst was observed is far from hydrostatic equilibrium.

The energy of emitting electrons must be high to prevent the source decay during the entire pulse. The lower bound of

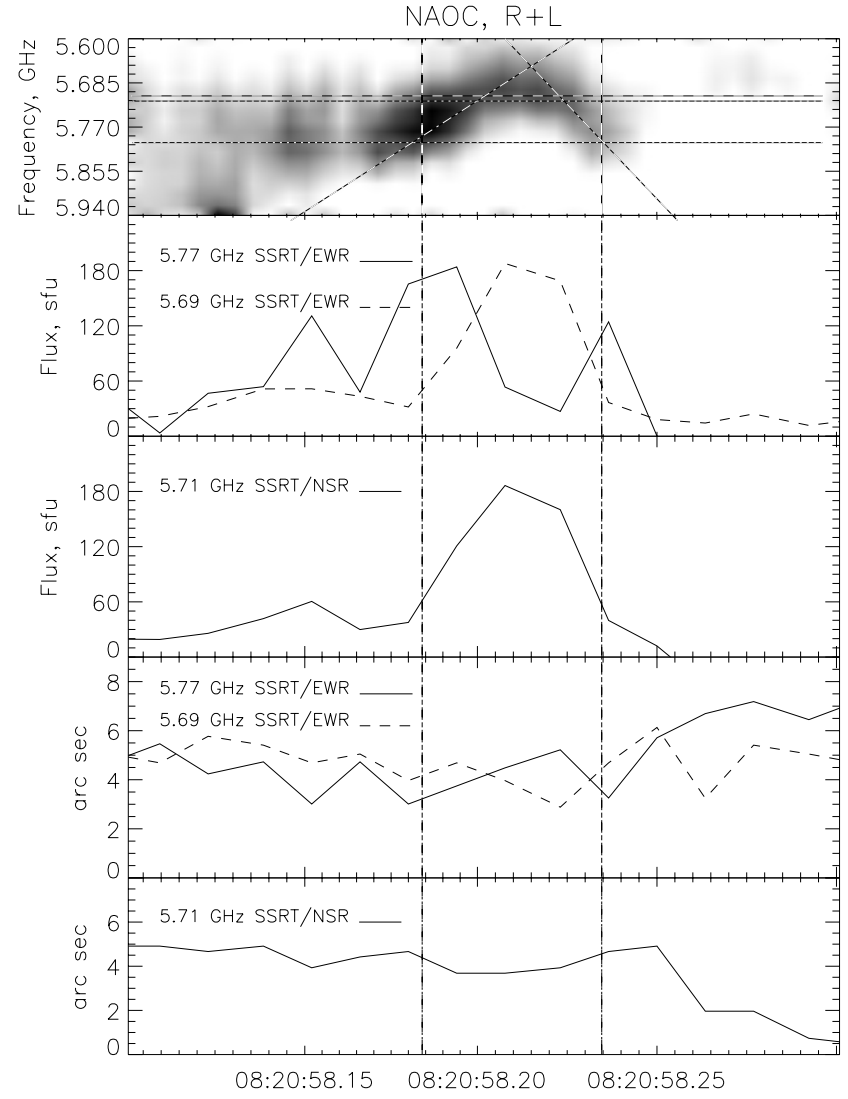

Fig. 8. September 17, 2001. Spectral and temporal characteristics of the subsecond pulse.

their velocity $v_{\text {acc }}$ can be inferred from the condition that the time of Coulomb scattering from background plasma ions $t_{D}$ must exceed the total duration of the U-structure (up to $0.5 \mathrm{~s}$ ), where $t_{D}=3.1 \times 10^{-20} \frac{v_{\text {acc }}^{3}}{n}(\mathrm{~s})$. Thus $v_{\text {acc }}>1.5 \times 10^{10} \tau_{\text {tot }}^{1 / 3} \mathrm{~cm} / \mathrm{s}$, or $v_{\text {acc }}>(0.8-1.2) \times 10^{10} \mathrm{~cm} / \mathrm{s}$. Note that in the case of emission at the fundamental frequency, plasma density must be higher and the condition on the value of the energy of emitting electrons becomes still more rigorous.

The upper estimates of the exciter velocity range is $\frac{\lambda}{\cos \theta \tau_{\mathrm{sst}}} \approx \frac{3 \times 10^{8}}{0.85 \tau_{\mathrm{sst}}}=(0.16-0.9) \times 10^{10} \mathrm{~cm} / \mathrm{s}$, which is lower than the estimate of the electron beam velocity $v_{\text {acc }}$. Here $\theta$ is the pitch angle of the beam electrons. In this formula we take the pitch angle $\theta=30^{\circ}$.

It should also be noted that no polarization reversal for different branches of the U-structure is observed in all cases that were studied. Thus spatially resolved observational data are difficult to reconcile with the interpretation of the U-shaped bursts as the emission of the beam of accelerated electrons traveling along a closed magnetic loop.

\subsection{MHD disturbances}

Let us consider another possibility: that U-shaped structures are produced by the plasma response of a pressure and density adjustment to a local heating pulse in a part of flare loop with the size of about $\lambda$. Such disturbances can appear due to local impulsive heating of this part of the loop. Observations in 


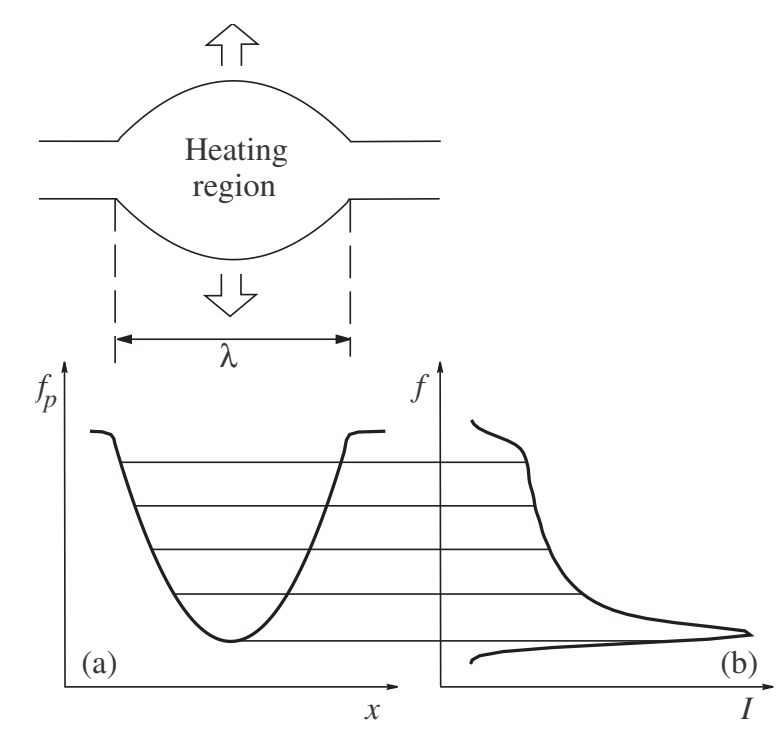

Fig. 9. Sketch of the heating region and of the spectrum shape dependence (b) on the density distribution (a).

hard X-rays show fast pulses with the rise and decay times as short as $20 \mathrm{~ms}$ and durations as short as $45 \mathrm{~ms}$ (Kiplinger et al. 1983). It is evident that the temporal convolution of the electron acceleration process with electron propagation effects will lead to a delay and spread in the hard X-ray burst time profile, even for an instant injection of electrons. The hypothesis of rapid local heating has been discussed since the Skylab mission (e.g. Matler 1978; Dulk et al. 1979; Ramaty et al. 1980).

The existence of local heating areas in the flaring loops is confirmed by soft X-rays observations. (Acton et al. 1992; Feldman et al. 1994; Doschek 1999; Kovalev et al. 2001). Images obtained by Yohkoh and SOHO show that the hottest portion of the soft X-ray flare is located in compact regions at the top of flare loops. These compact regions form at, or shortly after, flare onset. The compact regions are only a few thousand kilometers in size and are small compared to the lengths of flaring loops. They believe this is inconsistent with the smoother intensity distribution along the loops expected from the models of chromospheric evaporation.

The plasma density and temperature are estimated as $10^{10}$ $10^{12} \mathrm{~cm}^{-3}$ and $(15-20) \times 10^{6} \mathrm{~K}$ respectively. Such structures may be the sources of narrowband microwave emission that is studied in this work. The only difference that should be noted is the lifetime of these regions: existing telescopes can detect only structures with duration of no less than about one minute, whereas type U-bursts are much shorter.

The increase in pressure in the heated part of the loop must lead to an increase of its cross-section and a decrease of the magnitude of the magnetic field. As a consequence of the plasma freezing-in to the magnetic field, plasma density decreases proportionately and, hence, the emission frequency decreases, as observed during the first branch of the U-structure. The second branch corresponds to the return compression due to the elasticity and the cooling of plasma.

To consider this effect we first neglect the thermal conductivity along the loop. The minimum frequency of the U-structure corresponds to a minimum density $n_{1}=n_{0}-\Delta n$, where $n_{0}$ is the plasma density prior to the impulsive heating. If after the heating the temperature $T_{1}=\alpha T_{0}$, then using the pressure balance equations across the magnetic loop $n_{0} T_{0}+\frac{\left(B_{0}\right)^{2}}{8 \pi}=n_{1} T_{1}+\frac{\left(B_{1}\right)^{2}}{8 \pi}$ and the condition of freezing-in $\frac{B_{0}}{n_{0}}=\frac{B_{1}}{n_{1}}$, it is possible to estimate the density variation as $\Delta n \approx n_{0} \frac{\beta}{2}(\alpha-1)$, where $\beta=\frac{8 \pi n_{0} T_{0}}{B_{0}^{2}}$. For $B_{0}=100 \mathrm{G}$ and $T_{0}=2 \mathrm{MK}$ we obtain $\beta=0.08$. Observations provide $\Delta n / n=$ 0.2 , which gives a reasonable estimate of the value of $\alpha \leq 6$ or $T_{1} \leq 12 \mathrm{MK}$. Such temperatures are typical of flares and, hence, the observed decrease in plasma density may well be explained by the processes of local heating of plasma.

If the rate of plasma pressure equalizing along the flaring loop is about the velocity of sound $c_{\mathrm{s}}=1.51 \times 10^{4} \sqrt{T(\mathrm{~K})} \mathrm{cm} / \mathrm{s}$, then the settling time of the equilibrium distribution of density is on the order of $\tau=\lambda / c_{\mathrm{s}}$. With the scale $\lambda=3$ thousand kilometers, the time is several seconds, or significantly larger than the observed duration of the type $U$ structures in the microwave range.

A rapid cooling of plasma heated in a part of the magnetic loop must be brought about by the escape of hot electrons along the magnetic field. According to Ivanov et al. (1969), the expansion of hot plasma along a magnetic loop has the character of a wave. An estimate of the expansion front velocity was obtained by Bardakov (1985) $V_{\mathrm{fr}} \approx(m / M)^{1 / 4}\left(n_{0} / n_{1}\right)^{1 / 2} V_{T_{1}}$, where $V_{T_{1}}$ is the thermal velocity of hot electrons, $m / M$ is the electron to ion mass ratio, and $n_{0} / n_{1}$ are the plasma densities inside and outside of the region with the heated plasma. When $n_{0} / n_{1} \approx 1$ and $T_{1}=12 \mathrm{MK}$, the velocity of the front is about $2 \times 10^{8} \mathrm{~cm} / \mathrm{s}$ and the characteristic time of plasma cooling is about one second for the length $\lambda=3 \times 10^{3} \mathrm{~km}$.

The influence of the cooling along the loop on its transverse expansion depends on the relation between the transverse and longitudinal dimensions of the heating region. The radius of the heated loop can be estimated as the product of the Alfven velocity $c_{\mathrm{A}}=2.03 \times 10^{11} \mathrm{~B} / \sqrt{n}$ by one half of the time interval $\tau_{\text {ssrt }}$ between the recordings of two branches of the U-structure. For $B=100 \mathrm{G} c_{\mathrm{A}} \approx 6.3 \times 10^{7} \mathrm{~cm} / \mathrm{s}$, and we have the estimate of the loop radius of about $30 \mathrm{~km}$ for $\tau_{\mathrm{ssrt}}=100 \mathrm{~ms}$. This radius is considerably smaller than $\lambda$, and the transverse expansion and the decrease in density will be important.

Kovalev et al. (2001) obtained for the cooling time of the coronal sites of local heating the value of about $0.4 \mathrm{~s}$ and it is close to the duration of type U-bursts.

Thus, local heating can provide a plasma density disturbance corresponding to the observed dynamical and spatial features of the U-bursts. From the above estimates of the source size we can calculate the brightness temperature for $1 \mathrm{sfu}$ flux as $5.5 \times 10^{9} \mathrm{~K}$. In the last event with the flux of 180 sfu the temperature is up to $10^{11} \mathrm{~K}$, and such a value can be provided only by coherent emission mechanisms. The energy that is needed for a growth of plasma turbulence can be provided by accelerated electrons existing in the loop before the local plasma heating, or appearing during this process. The instability responsible for the plasma wave amplification can be either a beam-like or loss-cone instability. Investigation of this process is beyond the scope of the present paper. 
Emission can be triggered by the same process of plasma heating. The observed growth time of emission is about $10 \mathrm{~ms}$, so taking into account the process of conversion of plasma turbulence to the transverse electromagnetic wave we can estimate the instability growth rate, producing plasma turbulence, as $10^{4}$ $\mathrm{s}^{-1}$. Before the heating this value can be too low in comparison with the Coulomb collision frequency at the temperature of $2 \mathrm{MK}$ and the density of $10^{11} \mathrm{~cm}^{-3}$, and the instability is suppressed. This conclusion does not depend on the type of instability.

The plasma density in the hot region varies over the range from $n_{0}-\Delta n$ to $n_{0}$; however, the contribution of the various sections of this interval to radiation is different, which results in the shaping of a narrow spectral band of emission. Firstly, the conditions for generation of radiation are most favorable in the middle of the hot region. The higher temperature of plasma reduces the threshold of instability development. Also, the gradients of a magnetic field, of plasma density, and of temperature are minimal there. The large gradients make the development of instabilities more difficult due to the rapid escape of accelerated particles from the resonance with the growing plasma oscillations.

Secondly, the instantaneous spectrum with a relatively narrow bandwidth can be formed simply as the result of the density distribution. Figure 9 is a scheme of the emission region where plasma density varies as a parabolic law. Even if the emission power from each cross-section is the same, the entire radiation spectrum has a distinct maximum corresponding to the density in the middle of the hot region where the density gradient is minimal. Assuming the disturbance amplitude $\Delta n / n_{0}=0.2$ and the emission mechanism with the relative intrinsic bandwidth of $1 \%$, we have the total spectrum with a relative bandwidth no more than $3 \%$, or about $180 \mathrm{MHz}$ in the case. This value is in agreement with observational results.

\section{Conclusions}

The displacements of the position of the sources referring to different branches of the microwave U-structure do not exceed three thousand kilometers. This is in conflict with the assumption that the change of the emission frequency of narrow band microwave bursts is associated with the propagation of the electron beam along the flare loop.

The observed dynamics of microwave emission fits well with the concepts of the magnetohydrodynamic response to impulsive heating of a limited part of the magnetic loop with the diameter of several tens of kilometers and with the length of about a few thousand kilometers. Coherent emission appears after the excitation of Langmuir turbulence by electron beams existing in the loop or that were accelerated during the heating. The plasma heating determine the plasma density variation, and the entire bandwidth of the microwave pulse as a result. The instantaneous bandwidth of the pulse can be determined by the form of plasma density distribution along this local region.
Acknowledgements. We are grateful to the referee for valuable suggestions which greatly improved this paper, Dr. S. Lesovoi for supplying us with the SSRT data on subsecond pulses, Dr. V. Grechnev for his help in aligning the SSRT map with images in the other emissions and useful discussions, and to Mr. Mikhalkovsky for his assistance in preparing the English version of the manuscript. The X-ray data used here became available to us thanks to efforts of colleagues operating the Yohkoh missions and the Nobeyama Radioheliograph. This work was supported by Russian projects of RFBR Nos. 02-02-39030, and 03-02-16229, Vuz grant (E02-3.2-489), Integratsiya project No. I0208, and Astronomiya and Chinese grant G2000078403 and NSFC.

\section{References}

Acton, L. W., Feldman, U., Bruner, M. E., et al. 1992, PASJ, 44, L71 Altyntsev, A. T., Grechnev, V. V., Konovalov, S. K., et al. 1996, ApJ, 469, 976

Altyntsev, A. T., Lesovoi S. V., Meshalkina N. S., \& Sych R. A. 2003, A\&A, 400, 337

Aschwanden, M. J. 2002, Space Sci. Rev., 101, 1

Bardakov, V. M. 1985, Soviet J. Plasma Phys., 11, 699

Bruggmann, G., Benz, A. O., Magun, A., \& Stehling, W. 1990, A\&A, 240,506

Caroubalos, C., Poquerusse, M., Bougeret, J.-L., \& Crepel, R. 1987, ApJ, 319, 503

Correia, E., Costa, J. E. R., Kaufmann, P., Magun, A., \& Herrmann, R. 1995, Sol. Phys., 159, 143

Doschek, G. A. 1999, ApJ, 527, 426

Dulk, G. A., Melrose D. B., \& White S. M. 1979, ApJ, 234, 1137

Dulk, G. A. 1985, ARA\&A, 23, 169

Feldman, U., Hiei, E., Phillips, K. J. H., Brown, C. M., \& Lang, J. 1994, ApJ, 421, 843

Fleishman, G. D., \& Melnikov, V. F. 1998, Phys. Uspekhi, 41, 1157

Fu, Q., Qin, Z., Ji, H.,\& Pei, L. 1995, Sol. Phys., 160, 97

Grechnev, V. V., Lesovoi, S. V., Smolkov, G. Ya., et al. 2003, Sol. Phys., in press

Ivanov, A. A., Kozorovitsky, L. L., \& Rusanov B. D. 1969, Dokl. AN USSR 184,811

Kiplinger, A. L., Dennis, B. R., Emslie, A. G., Frost K. J., \& Orwig, L. E. 1983, ApJ, 265, L99

Kovalev, V.A., Chernov, G.P., \& Hanaoka, I. 2001, Astron. Lett., 27, 267

Lim, J., White, S. M., Kundu, M. R., \& Gary, D. E. 1992, Sol. Phys., 140,343

Matler C., Bai T., Crannell C. J., \& Frost K. J. 1978 ApJ, 223, 1058

Maxwell, A., \& Swarup, G. 1958, Nature, 181, 36

Melrose, D. V. 1987, Sol. Phys., 111, 89

Meshalkina, N. S., Altyntsev, A. T., Grechnev, V. V., Sych, R. A., \& Yan Yihua 2002, The 10th Europ. Solar Physics Meet., 1, 343

Ramaty, R. Colgate, S. A., Dulk, G. A., et al. 1980, Energetic Particles in Solar Flares, Chap. 4 in Solar Flares, ed. P. A. Sturrock, 117

Smolkov, G., Ya., Pistolkors, A.,A., Treskov, T. A., et al. 1986, Ap\&SS, 119, 1

Sych, R. A., Altyntsev, A. T., Grechnev, V. V., Meshalkina, N. S., \& Yan Yihua 2002, The 10th European Solar Physics Meeting, 2, 761

Wang, M., Fu, Q., Xie, R. X., Huang, G. L., \& Duan, C. C. 2001, A\&A, 380, 318

Wild, J. P., \& Smerd, S. F. 1972, ARA\&A, 10, 159 